\title{
The Effect of Eye Exercises of Acupoints on Myopia Progression: A 3-Year Cohort Report from the Beijing Myopia Progression Study
}

This article was published in the following Dove Press journal: Risk Management and Healthcare Policy

\author{
Yuan Bo Liang ${ }^{1,2}$ \\ Balamurali Vasudevan ${ }^{3}$ \\ Zhong Lin ${ }^{1,2}$ \\ Hong Jia Zhou ${ }^{1,2}$ \\ Kenneth J Ciuffreda ${ }^{4}$ \\ 'Eye Hospital and School of \\ Ophthalmology and Optometry, \\ Wenzhou Medical University, Wenzhou, \\ Zhejiang, People's Republic of China; \\ ${ }^{2}$ National Clinical Research Center for \\ Ocular Diseases, Wenzhou, Zhejiang, \\ People's Republic of China; ${ }^{3}$ College of \\ Optometry, Mid Western University, \\ Glendale, AZ, USA; ${ }^{4}$ Department of \\ Biological and Vision Sciences, SUNY \\ College of Optometry, New York, \\ NY, USA
}

Purpose: To investigate the impact of eye exercises of acupoints on myopic progression among Chinese urban students.

Methods: The Beijing Myopia Progression Study (BMPS) was a three-year cohort study, with 386 students (aged 6-17 years) enrolled at baseline. These students were invited to be reexamined in the years 2011, 2012, and 2013. The student's cycloplegic refraction at each visit and the parental non-cycloplegic refraction at baseline were performed. Students were also required to complete the eye exercise of acupoints questionnaire and the convergence insufficiency symptom survey (CISS).

Results: At the final follow-up, 226 students (57.2\%) with complete refraction and eye exercises questionnaire data were enrolled in the present study. In the multivariate analysis, only students who performed the eye exercises twice or more per day had less myopic refractive change $(\beta=0.32, p=0.04)$, as compared to those who performed the eye exercises less than twice per day. No significant association was found between the CISS score and the refractive change for items in the eye exercises questionnaire.

Conclusion: In this study cohort, the Chinese eye exercises of acupoints had a modest effect on reducing myopic progression among Chinese urban students aged 6 to 17 years. The precise mechanism remains unclear, especially in the absence of a control group.

Keywords: eye exercises, acupoints, myopia, progression, myopia risk factors, convergence insufficiency symptom survey

\section{Introduction}

The Chinese eye exercises of acupoints have been a mandatory measure among Chinese students (approximately ages from 6 to 17 years) before college, as introduced by the Chinese National Education Commission. These are bilateral, acupoint, self-massage exercises, performed twice a day per government requirement ( 5 mins in the morning and 5 mins in the afternoon) during each school day, with an aim to relieve ocular fatigue and reduce myopia.

Several studies have reported that these exercises may have an effect for myopia prevention and/or visual acuity improvement in Chinese school students. ${ }^{1-4}$ One study indicated that the local ocular blood circulation among Chinese myopic teenagers $(n=48)$ was improved after performing the eye exercises as assessed by color Doppler imaging. ${ }^{5}$ In a randomized, double-blind controlled trial in school children of Anyang, central China, Li et al reported that those who performed the eye exercise conventionally had greater alleviation of accommodative lag than
Correspondence: Zhong Lin PhDEye Hospital and School of Ophthalmology and Optometry,

Wenzhou Medical University, No. 270

West College Road, Wenzhou, Zhejiang

325027, People's Republic of China

Tel +86-577-88824II6

Email linzhong_@126.com
Risk Management and Healthcare Policy 2020:13 2793-2799

mit your manuscript

DovePres: $f$ in $\square$ 
those who either massaged the sham acupoints or simply closed their eyes. ${ }^{6}$ Later, in a 2-year longitudinal study from the same population, the authors reported that children who performed the eye exercises well had a slightly lower, but not significant, myopic progression $(0.15 \mathrm{D})$ than the children who did not perform the exercise. ${ }^{7}$

The Beijing Myopia Progression Study (BMPS) is a 3-year cohort study that aims to investigate the risk factors for refractive error change in Chinese urban students. The baseline data of the BMPS reported that students who performed the eye exercises seriously and according to the instructions, and who were acquainted with the acupoints, seemed to manifest less ocular fatigue symptoms. ${ }^{8}$ However, the effect on reducing myopia was not assessed. ${ }^{8}$ Hence, the aim of the present study was to investigate the impact of the eye exercises of acupoints on myopia progression derived from the longitudinal data of BMPS.

\section{Methods}

\section{Subjects}

The study design, procedures, and baseline characteristics of BMPS have been reported elsewhere. ${ }^{9}$ Briefly, children (aged 6-17 years) from primary and secondary schools in Beijing were recruited from July to September 2010. The inclusion criteria were: (1) best-corrected visual acuity (BCVA) 0.1 (log minimum angle of resolution, LogMAR) or better; and (2) willing to cooperate and return for scheduled visits. The exclusion criteria were: (1) presence of amblyopia and/or strabismus; (2) history of intraocular surgery or penetrating ocular trauma; and (3) severe medical/ocular health problems. The main vision examination included ocular biometry, cycloplegic refraction, and the eye exercises of acupoints questionnaire. The parents of these students were also invited to join the study. Subsequently, the enrolled students were invited to be reexamined at a similar time of the year in 2011, 2012, and 2013.

The study followed the tenets of the Declaration of Helsinki and was approved by the Beijing Tongren Hospital Ethics Committee. All participants (children and their parents) signed a written informed assent and consent, respectively.

\section{Eye Exercises of Acupoints Questionnaire} The participants were asked to complete a vision selfdesigned eye exercises questionnaire. Details of the questionnaire were reported elsewhere. ${ }^{8}$ Briefly, it consisted of 11 items related to motivation, performance frequency, and attitude towards the eye exercises of acupoints.

\section{Convergence Insufficiency Symptom Survey (CISS)}

The participants were asked to complete a symptom questionnaire. Details of the questionnaire were reported elsewhere. ${ }^{10,11}$ Briefly, the validated CISS consists of 15 items with 5 response categories for each item. ${ }^{10}$ It is scored as follows: never (0), infrequently (1), sometimes (2), fairly often (3), and always (4) with regard to visionrelated symptoms. The total score is the sum of scores for all 15 items (range from 0 to 60 ).

\section{Refractive Error}

All students received a cycloplegic autorefraction (Accuref-K9001, Shin Nippon, Japan) during each visit, whereas the parents only received a non-cycloplegic autorefraction (Accuref-K9001, Shin Nippon, Japan) during the baseline eye examination. Cycloplegic autorefraction was performed 20 mins after instilling three drops of cyclopentolate 1\% (Cyclogyl, Alcon). Three readings were obtained in each eye and averaged in all participants.

\section{Definitions}

Spherical equivalent (SE) refractive error was calculated as the sphere $+1 / 2$ cylinder. Due to the high correlation of the cycloplegic SE between the right and left eyes (Pearson correlation coefficient $0.95, \mathrm{p}<0.001$ ), only the SE of the right eye of each student was used in the analysis. Myopia was defined as SE $<-0.5$ diopters (D). Parental refractive error was defined as the average of the non-cycloplegic SE for the combined father and mother. ${ }^{12}$ Children's total refractive change was defined as the difference between the cycloplegic SE at the final follow-up minus the cycloplegic SE at baseline.

\section{Data Analysis}

The normally distributed parameters were presented as the mean \pm one standard deviation. The Student's $t$-test (or one-way analysis of variance, ANOVA) and the chi-square test were performed for comparison of the normal continuous data and the classified categorized data, respectively.

Univariate regression analyses for the association between the refractive change/CISS score and items of the eye 
exercises of acupoints, and possible myopia risk factors, were performed. In the multivariate regression analysis, items of the eye exercises of acupoints, age, gender, and significant factors in the univariate analyses were adjusted for the association with refraction change/CISS score using generalized linear models (GLMs). Univariate and multivariate (after adjusting for the same confounders) relative risk (RR) and the $95 \%$ confidence interval (CI) for newly-developed myopia involving the different items of the eye exercises of acupoints questionnaire were performed using GLMs.

\section{Results}

Three hundred and ninety-five students with a completed eye examination, including cycloplegic refraction, and the eye exercises of acupoints questionnaire at the baseline study of BMPS in the year 2010, were required to be reexamined in 2011, 2012, and 2013. The mean follow-up time was $35.2 \pm 2.5$ months. Two hundred and twenty-six students with complete questionnaire and refractive data were enrolled for this study. There were 132 primary students (58.4\%) and 94 secondary students (41.6\%), including 111 boys (49.1\%) and 115 (50.9\%) girls. The three-year cycloplegic refractive change was $-1.46 \pm 1.01 \mathrm{D}$ in these 226 students. The refractive change was $-1.53 \pm 0.99 \mathrm{D}$ and $-1.40 \pm 1.03 \mathrm{D}$ in the boys and girls $(\mathrm{p}=0.34)$, respectively.

Table 1 presents the 3 year refractive change and the associations between the refractive change and each item of the eye exercises of acupoints questionnaire. The refractive change was significantly more myopic in the students who were acquainted with the acupoints as compared to those who were not $(-1.65 \pm 0.91$ vs $-1.34 \pm 1.06, \mathrm{p}=0.03)$. The univariate regressions for refractive change and different items of the eye exercises questionnaire also showed that students who were acquainted with the acupoints tended to have more myopic refractive change $(\beta=-0.31, p=0.03)$, as compared to those who were not or only moderately acquainted with the acupoints. Furthermore, students whose eye exercises were taught by their teachers, doctors, or healthy counselors also tended to have a more myopic refractive change $(\beta=-0.36, p=0.057)$, as compared to those whose

Table I The Refractive Change and Associations Between Refractive Change and Each Item of the Eye Exercises of Acupoints Questionnaire at Baseline

\begin{tabular}{|c|c|c|c|c|}
\hline & Number & $\begin{array}{l}\text { Three-Year Refractive } \\
\text { Change }\end{array}$ & $\begin{array}{l}\text { Univariate } \beta \text { Coefficient } \\
\text { ( } p \text { value) }\end{array}$ & $\begin{array}{l}\text { Multivariate } \beta \text { Coefficient } \\
\text { ( } p \text { value) }{ }^{b}\end{array}$ \\
\hline $\begin{array}{l}\text { Times per day (in school) } \\
\quad<2 \\
\geq 2\end{array}$ & $\begin{array}{l}39 \\
187\end{array}$ & $\begin{array}{l}-1.72 \pm 1.10 \\
-1.40 \pm 0.98\end{array}$ & $0.33(0.07)$ & $0.32(0.04)$ \\
\hline $\begin{array}{l}\text { Seriously done times per week } \\
\text { None } \\
\quad<3 \\
\geq 5\end{array}$ & $\begin{array}{l}22 \\
44 \\
160\end{array}$ & $\begin{array}{l}-1.26 \pm 1.11 \\
-1.41 \pm 1.02 \\
-1.47 \pm 0.99\end{array}$ & $-0.09(0.39)$ & $-0.02(0.83)$ \\
\hline $\begin{array}{l}\text { Eye exercises were taught by } \\
\text { Atlas/Classmate } \\
\text { Teacher/Doctor/healthy counselor }\end{array}$ & $\begin{array}{l}35 \\
191\end{array}$ & $\begin{array}{l}-1.13 \pm 0.88 \\
-1.50 \pm 1.02\end{array}$ & $-0.36(0.057)$ & $-0.12(0.47)$ \\
\hline $\begin{array}{l}\text { Speed } \\
\text { Faster/Slower than the broadcast/ } \\
\text { At will } \\
\text { Following the broadcast }\end{array}$ & $\begin{array}{l}37 \\
189\end{array}$ & $\begin{array}{l}-1.63 \pm 0.99 \\
-1.43 \pm 1.02\end{array}$ & $0.20(0.28)$ & $0.12(0.46)$ \\
\hline $\begin{array}{l}\text { Acquaintance of the acupoints } \\
\text { No/Moderate } \\
\text { Yes }\end{array}$ & $\begin{array}{l}133 \\
93\end{array}$ & $\begin{array}{l}-1.34 \pm 1.06 \\
-1.65 \pm 0.91^{\mathrm{a}}\end{array}$ & $-0.31(0.03)$ & $-0.20(0.09)$ \\
\hline $\begin{array}{l}\text { Perform additional eye exercises } \\
\text { (outside school) } \\
\text { No } \\
\text { Yes }\end{array}$ & $\begin{array}{l}151 \\
75\end{array}$ & $\begin{array}{l}-1.47 \pm 1.04 \\
-1.45 \pm 0.96\end{array}$ & $0.03(0.86)$ & $-0.06(0.64)$ \\
\hline
\end{tabular}

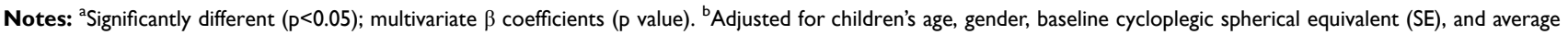
parental SE. Refractive change in diopters (D). 
Table 2 The Convergence Insufficiency Symptom Survey (CISS) Scores at Baseline and Their Associations with Refractive Change as a Function of Each Item of Eye Exercises of Acupoints Questionnaire at Baseline

\begin{tabular}{|c|c|c|c|c|}
\hline & $\mathbf{N}$ & CISS Score & $\begin{array}{l}\text { Univariate } \beta \text { Coefficient } \\
\text { ( } p \text { value) }\end{array}$ & $\begin{array}{l}\text { Multivariate } \beta \text { Coefficient } \\
\text { ( } p \text { value })^{c}\end{array}$ \\
\hline Total & 226 & $12.7 \pm 7.7$ & $0.04(<0.00 \mathrm{I})$ & $0.01(0.18)$ \\
\hline \multicolumn{5}{|l|}{ Times per day (in school) } \\
\hline$<2$ & 39 & $12.1 \pm 6.1$ & $0.06(0.049)$ & $0.02(0.37)$ \\
\hline$\geq 2$ & 187 & $12.7 \pm 7.7$ & $0.04(<0.00 \mathrm{I})$ & $0.01(0.18)$ \\
\hline \multicolumn{5}{|l|}{ Seriously done per week } \\
\hline None & 22 & $12.5 \pm 6.9$ & $0.05(0.18)$ & $0.01(0.77)$ \\
\hline$<3$ & 44 & $14.5 \pm 7.3$ & $0.04(0.06)$ & $0.01(0.58)$ \\
\hline$\geq 5$ & 160 & $12.1 \pm 7.5$ & $0.04(<0.001)$ & $0.01(0.29)$ \\
\hline \multicolumn{5}{|l|}{ Eye exercises were taught by } \\
\hline Atlas/Classmate & 35 & $12.6 \pm 7.2$ & $0.05(0.01)$ & $0.00(0.97)$ \\
\hline Teacher/Doctor/healthy counselor & 191 & $12.6 \pm 7.5$ & $0.04(<0.001)$ & $0.01(0.22)$ \\
\hline \multicolumn{5}{|l|}{ Speed } \\
\hline Faster/Slower than the broadcast/At will & 37 & $15.2 \pm 7.3$ & $0.03(0.26)$ & $0.02(0.50)$ \\
\hline Following the broadcast & 189 & $12.2 \pm 7.6^{* a}$ & $0.04(<0.001)$ & $0.01(0.23)$ \\
\hline \multicolumn{5}{|l|}{ Acquaintance of the acupoints } \\
\hline No/Moderate & 133 & $13.8 \pm 7.7$ & $0.03(0.005)$ & $0.00(0.83)$ \\
\hline Yes & 93 & $10.9 \pm 7.3^{b}$ & $0.04(0.001)$ & $0.02(0.10)$ \\
\hline \multicolumn{5}{|l|}{$\begin{array}{l}\text { Perform additional eye exercises (outside } \\
\text { school) }\end{array}$} \\
\hline No & 151 & $12.4 \pm 7.2$ & $0.04(0.002)$ & $0.01(0.24)$ \\
\hline Yes & 75 & $13.2 \pm 8.7$ & $0.04(<0.001)$ & $0.01(0.25)$ \\
\hline
\end{tabular}

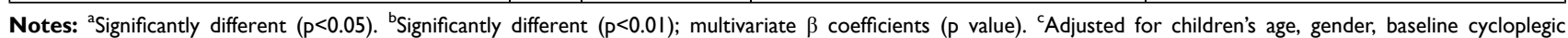
spherical equivalent (SE), and average parental SE.

eye exercises were learned from either the atlas or from classmates. However, after adjusting for the children's age, gender, baseline cycloplegic SE, and average parental SE, the effects of the two items were no longer significant. In the multivariate analysis, students who performed the eye exercises twice or more per day had less myopic refractive change $(\beta=0.32, p=0.04)$, as compared to those who performed the eye exercises less than twice per day, although it was only borderline significant in the univariate analysis $(\beta=0.33, p=0.07)$. Similar results were found for the two-year and one-year refractive changes. Both the two-year and oneyear refractive changes were significantly less myopic for students who performed the eye exercises twice or more per day, as compared to students who performed the eye exercises less than twice per day (two-year refractive change: $-0.95 \pm 0.77 \mathrm{D}$ vs $-1.28 \pm 0.84, \mathrm{p}=0.03$; one-year refractive change: $-0.48 \pm 0.53 \mathrm{D}$ vs $-0.73 \pm 0.59, \mathrm{p}=0.01$ ). There were also significant associations between the two-year/one-year refractive change and this item of the eye exercises questionnaire in the multivariate analysis (two-year: $\beta=0.34$, $p=0.01$; one-year: $\beta=0.24, p=0.01$ ). No significant results were found for the other items of the eye exercises questionnaire in the multivariate regression analysis.

Table 2 presents the CISS scores at baseline and their associations with the 3 year refractive change for each item of the eye exercises of acupoints questionnaire. The CISS score was significantly less in those students who followed the school broadcast when doing the exercises $(12.2 \pm 7.6$ vs $15.2 \pm 7.3, \mathrm{p}=0.03)$, and who were acquainted with the acupoints $(10.9 \pm 7.3$ vs $13.8 \pm 7.7$, $\mathrm{p}<0.01$ ), as compared to those who did not. Although the CISS score was associated with the refractive change for most items of the eye exercises questionnaire in the univariate analysis, none remained significant after adjusting for the children's age, gender, baseline cycloplegic SE, and average parental SE. There were also no significant associations between the two-year/one-year refractive change and the CISS score for each item of the eye exercises questionnaire in the multivariate regression analysis. 
There were 80 non-myopic children in the baseline eye examination, and 37 of them (46.3\%) had developed myopia in the final follow-up vision examination. Among these 80 students, a univariate logistic regression showed that students who performed the eye exercises twice or more per day had less risk for development of myopia (RR, 95\% CI: 0.56, 0.35-0.91), as compared to those who performed the eye exercises less than twice per day. However, after adjusting for children's age, gender, baseline cycloplegic SE, and average parental SE, no significant associations between items of the eye exercises of acupoints/CISS score and the new development of myopia were found (Table 3).

\section{Discussion}

The most important finding of this study was that children who performed these eye exercises more frequently per day had significantly less myopic progression. Less one-year, two-year, and three-year myopic progression was observed in children who performed the eye exercises twice or more per day as compared to those who performed them less, although it was not significant for the latter one. Furthermore, the multivariate analysis also consistently showed that children who performed the eye exercises more frequently had an effect on reducing the myopic progression. In our previous baseline study, no association was found between the prevalence of myopia and different aspects of the eye exercises. ${ }^{8}$ The reasons may be as follows. This hospital-based study tended to enroll urban students with more baseline myopic refraction. Furthermore, children with a more myopic refraction may have had greater determination, or were under greater psychological pressure, to do so to reduce their myopic progression. ${ }^{8}$ However, these same children were also very likely to be exposed to other high-risk factors for causation of myopia, such as increased near-work activity hours, less outdoor activity hours, a crowded living environment, and a higher probability of having myopic parents. ${ }^{13-15}$ Thus, the effect of the eye exercises on reducing myopic progression with 10-20 mins of self-massage time each day in this highly susceptible population suggests its cumulative effectiveness. This is also supported in a population of

Table 3 Relative Risk (RR) and 95\% Confidence Interval (Cl) for Students with Newly-Developed Myopia in Urban Students in Beijing

\begin{tabular}{|c|c|c|c|c|}
\hline & \multicolumn{2}{|c|}{ Univariate } & \multicolumn{2}{|c|}{ Multivariate $^{a}$} \\
\hline & $\mathbf{R R}$ & $95 \% \mathrm{Cl}$ & $\mathbf{R R}$ & $95 \% \mathrm{Cl}$ \\
\hline \multicolumn{5}{|l|}{ Times per day (in school) } \\
\hline$<2$ & Ref & & Ref & \\
\hline$\geq 2$ & 0.56 & $0.35,0.91$ & 0.80 & $0.53,1.22$ \\
\hline \multicolumn{5}{|l|}{ Seriously done per week } \\
\hline None & Ref & & Ref & \\
\hline$<3$ & 1.13 & $0.20,6.29$ & 2.04 & $0.99,4.20$ \\
\hline$\geq 5$ & 1.42 & $0.28,7.21$ & 1.52 & $0.84,2.74$ \\
\hline \multicolumn{5}{|l|}{ Eye exercises were taught by } \\
\hline Atlas/Classmate & Ref & & Ref & \\
\hline Teacher/Doctor/healthy counselor & 0.98 & $0.52,1.88$ & 0.73 & $0.4 I, I .31$ \\
\hline \multicolumn{5}{|l|}{ Speed } \\
\hline Faster/Slower than the broadcast/At will & Ref & & Ref & \\
\hline Following the broadcast & 1.18 & $0.53,2.62$ & 1.23 & $0.58,2.59$ \\
\hline \multicolumn{5}{|l|}{ Acquaintance of the acupoints } \\
\hline No/Moderate & Ref & & Ref & \\
\hline Yes & 1.09 & $0.68,1.75$ & 1.13 & $0.74,1.73$ \\
\hline \multicolumn{5}{|c|}{ Perform additional eye exercises (outside school) } \\
\hline No & Ref & & Ref & \\
\hline Yes & 1.29 & $0.77,2.15$ & 1.18 & $0.82,1.69$ \\
\hline CISS score & 0.97 & $0.94,1.01$ & 0.99 & $0.97,1.02$ \\
\hline
\end{tabular}

Note: ${ }^{a}$ Adjusted for children's age, gender, baseline cycloplegic spherical equivalent (SE), and average parental SE.

Abbreviation: Ref, reference group. 
rural children where children who performed the eye exercises seriously and frequently were less likely to develop myopia, after adjusting for the confounders. ${ }^{4}$

Although these exercises have been performed nationally for more than half a century, the possible mechanism for relieving ocular fatigue and reducing myopia is not clear. Based on the theory of Traditional Chinese Medicine (TCM), one possible mechanism of the eye exercise is believed to be the motivation in meridians and collaterals, thereby relieving eye strain and recovering eye function, after precise massage on acupuncture acupoints. ${ }^{16,17}$ From the perspective of modern medicine, massaging on the acupoints around the eyes will relax the intrinsic eye muscles and reduce eye fatigue. ${ }^{18}$ More importantly, it may also accelerate local blood circulation and consequently improve ocular metabolism, ${ }^{18,19}$ as it was reported that schoolchildren with myopia were more frequently diagnosed with cerebral venous hemodynamic dysfunction. ${ }^{20}$ This was supported by Doppler observation in which the peak systolic velocity of the central retina artery and the ophthalmic artery increased after the eye exercises. ${ }^{5}$ It was further supported by a randomized, placebocontrolled, cross-over study, where the blood flow velocity of the ophthalmic artery increased during needling visionrelated acupoints of traditional Chinese acupuncture. ${ }^{21}$ Our previous baseline study reported that students who performed the eye exercises seriously and according to the instructions, and who were acquainted with the acupoints, seemed to manifest a lower CISS score, ie, less ocular fatigue symptoms. ${ }^{8}$ However, no association between the CISS score and the progression of refractive error was found in the current longitudinal study. Hence, these two studies together suggested the impact of the eye exercises of acupoints on reducing myopia progression does not necessarily relieve near vision symptoms in these children. It should also be noted that simple eye closure to cease near work may also reduce the risk of myopia progression. ${ }^{22}$ Hence, a welldesigned randomized controlled trial (RCTs), including the ocular blood flow measurement, would be valuable to further assess these effects, and hopefully elucidate their mechanisms and avoid confounds.

Potential limitations of the current study remain. First, as mentioned above, this hospital-based study tended to enroll children with more myopic refraction. Hence, generalization of the results of this study to those with lower degrees of myopia may be different. Second, the relatively small sample size may affect the power to detect the effect of the other items of the eye exercises. Third, follow-up time was short. Fourth, although it was a longitudinal study, the lack of either a control or placebo group made the conclusion less robust. Hence, either school-based or population-based longitudinal studies with a larger sample size, as well as well-designed RCTs, are warranted.

\section{Ethics Approval and Consent to Participate}

The study followed the tenets of the Declaration of Helsinki and was approved by the Beijing Tongren Hospital Ethics Committee. Written, informed consent of participation was obtained from children's parents/guardians.

\section{Consent for Publication}

Written, informed consent for publication was obtained from children's parents/guardians.

\section{Acknowledgments}

The authors thank Dr. Xiao Xia Li (Zhengzhou Second People's Hospital), Dr. Yi Cao Zhang (Anyang Eye Hospital), Dr. Xiao Dong Yang (Nanjing Tongren Hospital), Dr. Qian Jia (Handan Eye Hospital), Dr. Yue Wu (Beijing Xuanwu Hospital, Capital Medical University), and Dr. Xiao Gu Cai (Beijing Tongren Hospital, Capital Medical University) for their invaluable assistance in the data collection.

\section{Funding}

The study was supported by Zhejiang Provincial Natural Science Foundation of China (LQ18H120004), the Research Startup Project of Wenzhou Medical University (No. 89213008), and the Beijing Science \& Technology Novel Star Program (2009B44).

\section{Disclosure}

The authors declare that they have no competing interests.

\section{References}

1. Zhong P, Wang K, Zhang C, Zhou B. [The epidemiological investigation of myopia in junior students]. Prac $J$ Med Pharm. 2004;21:543-545. Chinese.

2. Zhang D, Tang H. [Study on the effect of attitude towards the eye exercises on vision]. Chin J School Doctor. 2006;20:501-503. Chinese.

3. Liu C, Wang J, Guo H, Wang J. [A survey on prevalence of myopia and its influential factors in middle school students]. Modern Preventive Medicine. 2010;37:3047-3051. Chinese.

4. Lin Z, Vasudevan B, Fang SJ, et al. Eye exercises of acupoints: their impact on myopia and visual symptoms in Chinese rural children. BMC Complement Altern Med. 2016;16:349. doi:10.1186/s12906016-1289-4 
5. Lei J. [Observation of ocular haemodynamic change pre and post doing the eye exercises using color Doppler flood image]. Chinese J Ultrasound Diagnosis. 2004;5:446-447. Chinese.

6. Li SM, Kang MT, Peng XX, et al. Efficacy of chinese eye exercises on reducing accommodative lag in school-aged children: a randomized controlled trial. PLoS One. 2015;10:e0117552. doi:10.1371/journal.pone.0117552

7. Kang MT, Li SM, Peng X, et al. Chinese eye exercises and myopia development in school age children: a nested case-control study. $\mathrm{Sci}$ Rep. 2016;6:28531. doi:10.1038/srep28531

8. Lin Z, Vasudevan B, Jhanji V, et al. Eye exercises of acupoints: their impact on refractive error and visual symptoms in Chinese urban children. BMC Complement Altern Med. 2013;13:306. doi:10.1186/ 1472-6882-13-306

9. Lin Z, Vasudevan B, Liang YB, et al. Baseline characteristics of near work-induced transient myopia. Optom Vis Sci. 2012;89:1725-1733.

10. Borsting EJ, Rouse MW, Mitchell GL, et al. Validity and reliability of the revised convergence insufficiency symptom survey in children aged 9 to 18 years. Optom Vis Sci. 2003;80:832-838. doi:10.1097/ 00006324-200312000-00014

11. Rouse M, Borsting E, Mitchell GL, et al. Validity of the convergence insufficiency symptom survey: a confirmatory study. Optom Vis Sci. 2009;86:357-363. doi:10.1097/OPX.0b013e3181989252

12. Lin Z, Gao TY, Vasudevan B, et al. Generational difference of refractive error and risk factors in the handan offspring myopia study. Invest Ophthalmol Vis Sci. 2014;55:5711-5717. doi:10.1167/ iovs.13-13693

13. Wang SK, Guo Y, Liao C, et al. Incidence of and factors associated with myopia and high myopia in Chinese children, based on refraction without cycloplegia. JAMA Ophthalmol. 2018;136:1017-1024. doi:10.1001/jamaophthalmol.2018.2658
14. Lin Z, Vasudevan B, Ciuffreda KJ, Gao TY, Zhou HJ, Liang YB. Generational difference of axial length and its risk factors in Urban and Rural China. J Ophthalmol. 2019;2019:1607064. doi:10.1155/ 2019/1607064

15. Choi KY, Mok AY-T, Do C-W, Lee PH, Chan HH-L. The diversified defocus profile of the near-work environment and myopia development. Ophthalmic Physiological Optics. 2020;40:463-471. doi:10.1111/opo. 12698

16. Chen JR, Lu YJ, Huang Y, Lin RY. [Literature study on the regularity of acupoints selection for the treatment of Myopia]. J Traditional Chinese Medicine. 2011;16:1413-1416. Chinese.

17. Ji CY, Sun HW, Li H. [Clinical research on the law of acupoint-selecting for adolescent myopia treated by acupuncture]. Henan Traditional Chinese Med. 2016;9:1642-1646. Chinese.

18. Field T. Massage Therapy, Acupressure, and Reflexology Ch. 4. (American Psychological Association; 2009:43-52.

19. Aggarwala KRG. Ocular accommodation, intraocular pressure, development of myopia and glaucoma: role of ciliary muscle, choroid and metabolism. Medical Hypothesis, Discovery Innovation Ophthalmol J. 2020;9:66-70.

20. Iastrebtseva TA, Demidova TE, Polikarpova VE. [Role of cerebral hemodynamics in the mechanism of development of acquired myopia in schoolchildren]. Vestn Oftalmol. 2008;124:10-13. Russian.

21. Litscher G. Computer-based quantification of traditional chinese-, ear- and Korean hand acupuncture: needle-induced changes of regional cerebral blood flow velocity. Neurol Res. 2002;24:377-380. doi:10.1179/016164102101200177

22. Ong E, Ciuffreda KJ. Accommodation, Nearwork, and Myopia. Santa Ana, CA. 1997.
Risk Management and Healthcare Policy

\section{Publish your work in this journal}

Risk Management and Healthcare Policy is an international, peerreviewed, open access journal focusing on all aspects of public health, policy, and preventative measures to promote good health and improve morbidity and mortality in the population. The journal welcomes submitted papers covering original research, basic science, clinical \& epidemiological studies, reviews and evaluations, guidelines, expert opinion and commentary, case reports and extended reports. The manuscript management system is completely online and includes a very quick and fair peer-review system, which is all easy to use. Visit http://www.dovepress.com/testimonials.php to read real quotes from published authors. 